\title{
LIST OF PARTICIPANTS
}

N. Abu-el-Ata, Bureau des Longitudes, 77 Avenue Denfert Rochereau, 75014 Paris, France

K. Aksnes, Center for Astrophysics, 60 Garden Street, Cambridge, Massachusetts 02138 , U.S.A.

G. Antonacopoulos, Department of Astronomy, Patras, Greece

M. Antonacopoulos, Department of Mathematics, Patras, Greece

J. Baumgarte, Lehrstuhı A für Mechanik, Technische Universität, Pockelsstrasse 4, D-3300 Braunschweig, West Germany

D. Benest, Observatoire de Nice, Le Mont Gros, F-06007 Nice Cedex, France

D.G. Bettis, Munich

K.B. Bhatnagar, IA/47-C Ashok Vihar, Delhi-110052, India

R. Bien, Astronomisches Recheninstitut, Munchhofstrasse 12-14, 69 Heidel berg 1, West Germany

V.R. Bond, NASA Johnson Space Center, FM5, Houston, Texas 77058, U.S.A.

P. Bretagnon, (see Abu-el-Ata)

C. Brookes, Department of Mathematics, University of Aston in Birmingham, Gosta Green, Birmingham B4 7ET, U.K.

0 . Calame, CERGA, Avenue Nicolai Copernic, 06130 Grasse, France

J. Chapront, (see Abu-el-Ata)

T. Christides, Department of Theoretical Mechanics, University of Thessaloniki, Thessaloniki, Greece

H. Claes, Facultés Universitaires de Namur, Rempart de la Vierge 8, B-5000 Namur, Belgium

A. Deprit, Department of Mathematics, University of Cincinnati, Cincinnati, Ohio 45221 , U.S.A.

L. Duriez, Laboratoire d'Astronomie, 1 Impasse de l'Observatoire, :59000 Lille, France

R. Dvorak, Universitätssternwarte Graz, Universitätsplatz 5, 8010 Graz, Austria

B. Garfinkel, Department of Astronomy, Yale University, New Haven, Connecticut 06520 , U.S.A.

J.D. Hadjidemetriou, (see Christides)

D.C. Heggie, Department of Mathematics, University of Edinburgh, King's Buildings, Mayfield Road, Edinburgh, EH9 3JZ, U.K.

J. Henrard, (see Claes)

P. Herget, Cincinnati Observatory, Observatory Place, Cincinnati, Ohio 45208, U.S.A.

H.G. Hertz, 2301 E St. NW, Apartment A608, Washington, D.C. 20037, U.S.A.

S. Hughes, University of Leicester, Department of Astronomy and History of Science, University Road, Leicester, LE] 7RH, U.K.

G. Janin, ESOC, Robert-Bosch-Strasse 5, D-6100, Darmstadt, West 
Germany

A.H. Jupp, Department of Applied Mathematics, University of Liverpool, P.0. Box 147, Liverpoo7, L69 3BX, U.K.

D.G. King-Hele, Royal Aircraft Establishment, Farnborough, Hampshire, U.K.

H. Kinoshita, (see Aksnes)

Y. Kozai, Tokyo Astronomical Observatory, Tokyo, Japan 181

J.H. Lieske, Jet Propulsion Laboratory, 4800 Oak Grove Drive, Pasadena, California 91103, U.S.A.

V.V. Markellos, Department of Astronomy, The University, Glasgow, G12 8Q0, U.K.

J.J. Martinez Benjamin, University of Texas at Austin, Department of Aerospace Engineering and Engineering Mechanics, Austin, Texas 78712 U.S.A

P.J. Message, (see Jupp)

M. Michalodimitrakis, (see Christides)

M. Moons, (see Claes)

J.D. Mulholland, Department of Astronomy, 15.212 R.L. Moore Ha11, College of Natural Sciences, University of Texas at Austin, Austin, Texas 78712 , U.S.A.

C. Desterwinter, NSWC/DL Code OK-10, Dahlgren, Virginia 22448, U.S.A.

G. Ratier, Observatoire du Pic du Midi, 65200 Bagneres de Bigorre, France

W.J. Robinson, Department of Mathematics, University of Bradford, BD7 1DP, U.K.

E.A. Roth, (see Janin)

J.L. Sagnier, (see Abu-el-Ata)

J. Schubart, (see Bien)

P.K. Seidelmann, U.S. Naval Observatory, Washington, D.C. 20390 , U.S.A.

R. Sergysels, Ecole Polytechnique, Faculté des Sciences Appl iquées, Université Libre de Bruxelles, Avenue F.D. Roosevelt 50, 1050 Bruxelles, Belgium

A.T. Sinclair, Royal Greenwich Observatory, Herstmonceux Castle, Hailsham, Sussex, U.K.

N. Spyrou, Institute of Astronomy, Madingley Road, Cambridge, CB3 OHA, U.K.

D. Standaert, (see Claes)

V. Szebehely, (see Martinez Benjamin)

D.B. Taylor, (see Markel los)

J.P. Vinti, Massachusetts Institute of Technology, Building W91-202, Cambridge, Massachusetts 02139, U.S.A.

J. Waldvogel, Eidgenossische Technische Hochschule, Seminar für Angewandte Mathematik, Clausiusstrasse 55, 8006 Zurich, Switzerland

C.A. Williams, Department of Astronomy, University of South Florida, Tampa, Florida 33620, U.S.A.

C. Zagouras, Department of Mechanics, University of Patras, Patras, Greece

K. Zare, Farah Park, Afarin Street No. 19, Isfahan, Iran 